\title{
Hiponatremia por oxcarbacepina en esclerosis múltiple, un caso para tener en cuenta
}

\author{
Hyponatremia by oxcarbacepin in multiple sclerosis, a case to take account \\ Diego Beltrán ${ }^{1,}$, Mario Montoya $^{1}$ \\ ${ }^{1}$ Departamento de Medicina Interna, Universidad del Sinú, Seccional Cartagena
}

Cartagena, Colombia

\begin{abstract}
Resumen
La hiponatremia es uno de los efectos adversos en los pacientes tratados con oxcarbazepina. Se puede ver enmascarada por múltiples síntomas, atribuibles a otras enfermedades, pero siempre debemos tenerla en mente en un paciente con antecedente de ingesta de dicho medicamento. Muchos de los pacientes son asintomáticos; cifras inferiores a $120 \mathrm{mmol} / \mathrm{L}$ ponen de manifiesto síntomas de severidad, como los presentados en el paciente con esclerosis múltiple que mostraremos a continuación. La habilidad diagnóstica del clínico, por tanto, juega un rol fundamental.

Debido a esto, se expone un caso en el que una mujer tratada con oxcarbacepina, para una de las formas clínicas de esclerosis múltiple, llega a urgencias con manifestaciones de este trastorno electrolítico. Por esto se escoge el caso clínico atendido por los autores directamente. Se concluye que no es infrecuente esta reacción adversa y que hay que tenerla en cuenta a la hora de los diagnósticos diferenciales.
\end{abstract}

Palabras clave: hiponatremia, sodio, carbamacepina, esclerosis múltiple, efectos, parestesia, oxcarbazepine.

doi: http://dx.doi.org/10.22265/acnef.4.2.248

\begin{abstract}
Hyponatremia is one of the adverse effects in patients treated with oxcarbazepine. This can be seen masked by multiple symptoms which are simply attributable to other diseases, but we must always keep it in mind in a patient with a history of taking that medication. Many of patients are asymptomatic; Doses lower than $120 \mathrm{mmol} / \mathrm{L}$ show symptoms of severity which presented a patient with multiple sclerosis, which we will show below, so that diagnostic ability of clinicians plays a fundamental role.

This is why the case is exposed in which a woman treated with oxcarbazepine for one of the clinical forms of multiple sclerosis, arrives at emergency departament with manifestations of this electrolytic disorder. For this reason, the clinical case served by the authors is chosen directly.

It is concluded that this adverse reaction is not uncommon and that it must be taken into account in the differential diagnosis.
\end{abstract}

Key words: Hyponatremia, sodium, carbamacepine, multiple sclerosis, effects, paresthesia, oxcarbazepine.

doi: http://dx.doi.org/10.22265/acnef.4.2.248

226-232 doi: http://dx.doi.org/10.22265/acnef.4.2.248

*Correspondencia: Diego Leonardo Beltrán Garzón, diegoleonardo18@yahoo.com

Recibido: 16-06-17 • Aceptado: 19-07-17 • Publicado en línea: 27-07-17 


\section{Introducción}

$\mathrm{O}$ xcarbazepina es un compuesto con estructura química similar a la carbamazepina y, probablemente, un mecanismo de acción similar. Los efectos secundarios vistos con el uso de la oxcarbazepina son sedación, dolor de cabeza, mareos, erupción cutánea, vértigo, ataxia, náuseas, hiponatremia, y diplopía.

Con la excepción de la hiponatremia ${ }^{1}$, estos efectos secundarios parecen presentarse con moderada frecuencia en pacientes que toman carbamazepina.

La hiponatremia es debido, al menos en parte, al aumento de la capacidad de respuesta de los túbulos colectores a la hormona antidiurética. Se considera, además, que es una de las formas del síndrome de secreción inapropiada de hormona antidiurética (SIADH).

Los valores séricos de sodio por debajo de 125 $\mathrm{mEq} / \mathrm{L}$ surgieron durante los primeros meses en un $3 \%$ de los pacientes tratados con oxcarbazepina en los ensayos clínicos ${ }^{2}$. Los pacientes ancianos, especialmente aquellos, especialmente aquellos que toman medicamentos concomitantes, son significativamente más propensos a desarrollar hiponatremia. La hiponatremia, por lo general, se desarrolla gradualmente durante los primeros meses de tratamiento, lo que puede explicar por qué la mayoría de los pacientes son asintomáticos ${ }^{3}$.

\section{Objetivos}

- Exponer un caso de hiponatremia ocurrido por oxcarbacepina en una clínica en la ciudad de Cartagena, en el año 2016.

- Mostrar el efecto adverso que puede producir la oxacarbacepina. Este puede ser, incluso, más molesto que el beneficio que representa su uso.

\section{Descripción del caso clínico}

Paciente femenino de 41 años de edad, con antecedente de esclerosis múltiple y neuromielitis óptica diagnosticadas hace 3 años, en tratamiento actual con oxcarbacepina cada 8 horas, pregabalina cada 8 horas y azatioprina 50mg día. Ingresa a Clínica Cartagena del Mar por cuadro clínico de 1 día de evolución, consistente en fatiga, adinamia, mareos y dolor generalizado, asociado a episodios eméticos de contenido alimentario en número de 4 . No refiere fiebre ni otros síntomas acompañantes.

Al examen físico, se encontró una paciente alerta con palidez mucocutánea leve, con escleras anictéricas. Cuello simétrico, móvil, sin masas. Tórax simétrico, normoexpansible, doloroso a la palpación superficial de forma difusa con ruidos respiratorios claros, sin agregados. Ruidos cardíacos rítmicos de buen tono, sin soplos. Abdomen blando, depresible, no doloroso. Extremidades simétricas, eutróficas, sin edema. Neurológico: consciente y orientada sin alteraciones del lenguaje. Con amaurosis bilateral sin fotorreactividad pupilar. Fuerza muscular 5/5 en todas las extremidades, parestesias en 4 extremidades. Reflejos osteotendinosos conservados $++/++++$, con aumento del polígono de sustentación de forma moderada.

Los exámenes de laboratorio de ingreso revelan normalidad en cuanto a la línea roja, línea blanca y trombocitos, con ionograma de ingreso que reporta hiponatremia severa de $111 \mathrm{mmol} / \mathrm{L}$ y cifras de glicemia en 109 mmol/L (tabla 1).

Se realizó RMN cerebral para descartar otro tipo de etiologías causantes de los síntomas actuales, en la que sólo se evidenciaron lesiones hiperintensas periventriculares sugestivas de desmielinización, compatibles con esclerosis múltiple (figura 1). Considerando, por su tiempo de evolución y diagnóstico, que es de tipo recurrente-remitente. 
Tabla 1.

Laboratorios de ingreso

\begin{tabular}{|l|c|c|}
\hline \multicolumn{2}{|c|}{ Valores normales pKPO } \\
\hline Hemoglobina (gr/dl) & 13,5 & $11-16 \mathrm{gr} / \mathrm{dl}$ \\
\hline Hematocrito (\%) & 37,4 & $35-43 \%$ \\
\hline Eritrocitos (mm3) & 4.220 .000 & $4,000.000-5,400.000$ \\
\hline Volumen Corpuscular Medio (um3) & 84 & $80-97 \mathrm{um} 3$ \\
\hline Hemoglobina media Corpuscular (pg) & 30 & $26,5-36,5 \mathrm{pg}$ \\
\hline CHCM (gr/dl) & 32 & $31-38 \mathrm{gr} / \mathrm{dl}$ \\
\hline Leucocitos (mm3) & 6,100 & $5.000-10.000 \mathrm{~mm} 3$ \\
\hline Neutrofilos \% & $79 \%$ & $45-65 \%$ \\
\hline Eosinofilos \% & $4 \%$ & $1-5 \%$ \\
\hline Linfocitos \% & $17 \%$ & $30-40 \%$ \\
\hline Plaquetas (mm3) & 347.000 & $150.000-450.000 \mathrm{~mm}$ \\
\hline Sodio (mmol/l) & 111,5 & $135-148 \mathrm{mmol} / \mathrm{L}$ \\
\hline Potasio (mmol/l) & 4,1 & $3.5-5,2 \mathrm{mmol} / \mathrm{L}$ \\
\hline Cloro (mmol/l) & 81,2 & $98-107,6 \mathrm{mmol} / \mathrm{L}$ \\
\hline
\end{tabular}

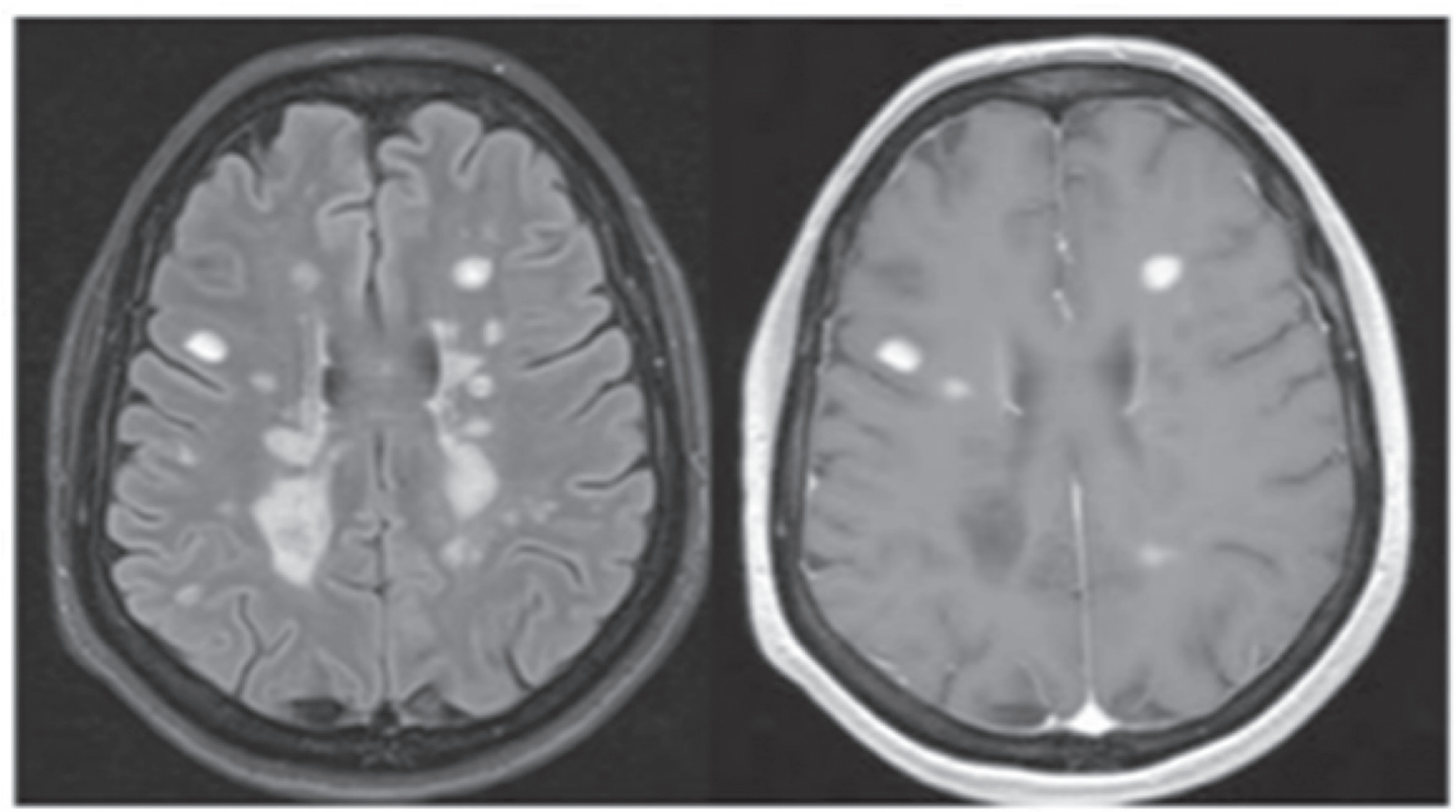

Figura 1. RMN cerebral inicial

228 Hiponatremia por oxcarbacepina en esclerosis múltiple, un caso para tener en cuenta 
Se inicia reposición de sodio desde el ingreso, con control posterior en $24 \mathrm{~h}$, en $120 \mathrm{mmol} / \mathrm{L}$ y disminución de los síntomas. Así mismo, se suspende ingesta de oxcarbacepina, pero se mantiene pregabalina y azatioprina como tratamiento base de la esclerosis múltiple en este caso.

Tras el tratamiento, 48 horas posingreso, obtenemos nuevo resultado de sodio, con mejoría comparativa a las cifras anteriores: $129 \mathrm{mmol} / \mathrm{L}$ (tabla 2). Ahora bien, en cifras seguras para la paciente, para no ocasionar sintomatología neurológica ni convulsiones.

Tabla 2.

Laboratorios de control

\begin{tabular}{|l|c|c|}
\multicolumn{2}{|c}{} & \multicolumn{2}{c|}{ Valores normales AHB } \\
\hline Sodio & 129,2 & $135-148 \mathrm{mmol} / \mathrm{L}$ \\
\hline Potasio & 3,9 & $3,5-5,2 \mathrm{mmol} / \mathrm{L}$ \\
\hline Cloro & 94,1 & $98-107,6 \mathrm{mmol} / \mathrm{L}$ \\
\hline
\end{tabular}

Paciente manifiesta sentirse estable, sin presentar nuevo episodio emético. Diuresis normal.

A las 24 horas se obtiene nuevo control de sodio sérico en: $135 \mathrm{mmol} / \mathrm{L}$ (tabla 3). Posteriormente, se procede a tomar niveles de sodio urinario, con valor en rango normal: $75 \mathrm{mmol} / \mathrm{L}$ en 24 horas.

Tabla 3.

Laboratorios complementarios

\begin{tabular}{|l|c|c|}
\multicolumn{2}{c}{ Valores normales } \\
\hline Creatinina & 0,8 & $0,9-1,3 \mathrm{mg} / \mathrm{dl}$ \\
\hline BUN & 15 & $7-18 \mathrm{mg} / \mathrm{dl}$ \\
\hline Sodio & 135 & $135-148 \mathrm{mmol} / \mathrm{L}$ \\
\hline Glicemia & 80 & $70-110 \mathrm{mg} / \mathrm{dl}$ \\
\hline
\end{tabular}

Así mismo, se proceden a realizar los otros paraclínicos complementarios para evaluar otras alteraciones, todos dentro de rangos aceptables en esta paciente.

Ante ausencia de síntomas, se procedió a alternancia de tratamiento de oxcarbacepina por tizanidina por sugerencia de neurología. No se obtienen resultados favorables dado que, con este tratamiento, reaparecen síntomas de esclerosis múltiple (alteración de la marcha, vértigo, parestesias y fatiga). Por lo anterior, decidimos suspender tizanidina y reiniciar oxacarbacepina paulatinamente, con media tableta en la mañana exclusivamente, evidenciando nuevamente descenso de niveles de sodio sérico a $128 \mathrm{mmol} / \mathrm{L}$ (tabla 4).

Tabla 4 Laboratorios control final

\begin{tabular}{|l|c|c|}
\multicolumn{2}{|c|}{ Valores normales AHB } \\
\hline Sodio & 128 & $135-148 \mathrm{mmol} / \mathrm{L}$ \\
\hline Potasio & 3,7 & $3,5-5,2 \mathrm{mmol} / \mathrm{L}$ \\
\hline Cloro & 93,4 & $98-107,6 \mathrm{mmol} / \mathrm{L}$ \\
\hline
\end{tabular}

Con esta prueba terapéutica, de reinicio de medicación, y con nuevo descenso del sodio, corroboramos nuestro diagnóstico inicial de hiponatremia secundaria a oxcarbacepina, ya que se mantuvieron siempre los otros medicamentos que venía tomando la paciente. Proponemos nueva alternancia de tratamiento por parte del servicio de neurología.

\section{Discusión}

El metabolismo de la oxcarbazepina se produce en el hígado, pero afecta mínimamente el sistema CYP. Esto representa una gran ventaja sobre la carbamazepina, en particular en pacientes que requieren múltiples fármacos, como el caso de nuestra paciente. La interacción medicamentosa y la pérdida del efecto de uno de los fármacos es poco probable por este hecho.

La hiponatremia asociada con la oxcarbazepina es debida, en parte, al aumento de la capacidad de respuesta de los túbulos colectores renales a la hormona antidiurética. Se considera, entonces, una de las formas del síndrome de secreción inapropiada de hormona antidiurética (SIADH). Aunque reportes tempranos habían sugerido que la 
oxcarbamazepina podía llegar a inducir liberación de vasopresina y provocar síndrome de secreción inapropiada de hormona antidiurética, otros estudios han desestimado este mecanismo ${ }^{3}$.

Otros estudios han documentado que, luego de la sobrecarga de agua, el sodio sérico y el clearence de agua libre disminuyeron en personas medicadas con oxcarbamazepina, sanas o enfermas, sin que hubiese en éstos últimos elevación de la vasopresinemia ${ }^{3}$.

Estos hallazgos indican que la hiponatremia inducida por oxcarbazepina no es del todo atribuible a un síndrome de secreción inadecuada de hormona antidiurética. Por ejemplo, vimos cómo los niveles de sodio en orina en nuestra paciente estaban en rango de normalidad (por lo general, en este síndrome están por encima de $40 \mathrm{mEq} / \mathrm{L}$ en una muestra simple de orina). Las cifras de potasio se mantuvieron en niveles normales ${ }^{4}$.

Existen algunos factores de riesgo para la hiponatremia por $\mathrm{OXC}$, como la edad mayor 40 años. Como lo demuestra la tasa más alta de la hiponatremia en mayores de 40 años, tiene una correlación más fuerte con tomar cualquier $\mathrm{OXC}$ (60 \%) o CBZ (20\%) 5. Esto mismo evidencia la edad de nuestra paciente, 41 años. Asimismo, se demuestra clínicamente en el caso que, a medida que se retiraba el medicamento y se volvía a instaurar de forma paulatina a modo de prueba terapéutica, los síntomas variaban proporcionalmente al medicamento, corroborando aún más el diagnóstico.

Es importante analizar este caso y tenerlo en cuenta en la práctica clínica de los médicos que manejen este tipo de medicamentos, pues la hiponatremia aguda puede causar edema cerebral, que puede conducir a convulsiones y encefalopatía, potencialmente mortales ${ }^{6}$. Debido a la adaptación cerebral, el grado de edema cerebral es menor con hiponatremia crónica y, en la mayoría de los pacientes, parece ser asintomática ${ }^{7}$ Asimismo, debemos mencionar, primero, que la reposición del sodio debe ser gradual en estos pacientes, dado el riesgo de mielinolisis pontina ${ }^{8}$; $\mathrm{y}$, en segundo lugar, que la suspensión del medicamento desencadenante debe ser el pilar principal de nuestro tratamiento, tal como se hizo desde un principio con nuestra paciente, según observamos.

\section{Conclusiones}

El personal de salud debe estar siempre atento a vigilar las interacciones y efectos adversos que se puedan ocasionar al administrar un fármaco. Balancear de forma lógica el riesgo/beneficio que la administración del fármaco representa para los pacientes. Asimismo, siempre tener en cuenta los diagnósticos diferenciales de este tipo de trastornos, que nos ayudan aún más a descartar otras etiologías. Este caso no es frecuente, pero se puede presentary, como vimos, si no se piensa no se diagnostica.

\section{Conflicto de intereses y financiación}

Artículo de carácter original e inédito. Los autores declaramos no tener ningún tipo de conflicto de interés. Así mismo, no reprodujimos ningún dato personal del paciente.

\section{Conflicto de intereses}

Los autores declaran no tener conflicto de intereses en la realización de este artículo.

\section{Responsabilidades éticas}

\section{Protección de personas y animales}

Los autores declaran que para esta investigación no se han realizado experimentos en seres humanos ni en animales. 


\section{Confidencialidad de los datos}

Los autores declaran que en este artículo no aparecen datos de pacientes.

Derecho a la privacidad y consentimiento informado

Los autores declaran que en este artículo no aparecen datos de pacientes.

\section{Contribución de los autores}

Diego Beltrán: autor principal y escritor del artículo. Mario Montoya: supervisión del artículo, obtención de bibliografia y médico tratante del caso asimismo facilitó la historia clínica. 


\section{Referencias}

1. Dong X, Leppik IE, White J, Rarick J. Hyponatremia from oxcarbazepine and carbamazepine. Neurology. 2005;65(12):1976-78. Disponible en: http://doi.org/10.1212/01.wnl.0000188819.45330.90

2. Glauser TA. Oxcarbazepine in the treatment of epilepsy. Pharmacotherapy 2001;21:904-19. Disponible en: https://doi.org/10.1592/ phco.21.11.904.34513

3. Sachdeo R, Wasserstein A, Mesenbrink J, D'Souza J. Effects of oxcarbazepine on sodium concentration and water handling. Ann Neurol. 2002; 51(5): 613-20. Disponible en: https://doi.org/10.1002/ana.10190

4. Food and Drug Administration. Highlights of Prescribing Information [Internet]. East Hanover: Novartis Pharmaceuticals Corporation. 2011. 34 p. Disponible en: http://www.accessdata.fda.gov/drugsatfda_docs/label/2014/021014s035,021285s030lbl.pdf

5. Schmidt D, Arroyo S, Baulac M, et al. Recommendations on the clinical use of oxcarbazepine in the treatment of epilepsy: a consensus view. Acta Neurol Scand. 2001;104(3):167-70. Disponible en: https://doi.org/10.1034/j.1600-0404.2001.00870.x

6. Berghuis B, de Haan G-J., van den Broek, MPH., Sander JW, Lindhout D, Koeleman, BPC. Epidemiology, pathophysiology and putative genetic basis of carbamazepine- and oxcarbazepine-induced hyponatremia. Eur J Neurol. 2016,23(9):139-9. Disponible en: https://www.doi.org/10.1111/ene.13069

7. Spasovski G, Vanholder, Allolio B, et al. Clinical practice guide on the diagnosis and treatment of hyponatremia. Eur J Endocrinol. 2014;170: L5-6. Disponible en https//:doi.org/doi: 10.1530/EJE-14-0393

8. Koch MW, Polman SK. Oxcarbazepine versus carbamazepine monotherapy for partial onset seizures. Cochrane Database Syst Rev. 2009;4:Art. No. CD006453. Disponible en: https://doi.org/10.1002/14651858.CD006453.pub2 